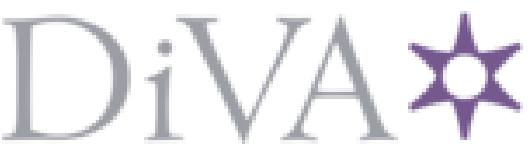

http://www.diva-portal.org

\title{
Postprint
}

This is the accepted version of a paper presented at Ninth International Model-Driven Requirements Engineering (MoDRE) workshop,September 24, 2019, Jeju Island, South Korea.

Citation for the original published paper:

Maksimov, Y V., Fricker, S A. (2019)

Framework for Analysis of Multi-Party Collaboration

In: 27th IEEE International Requirements Engineering Conference (RE 2019)

N.B. When citing this work, cite the original published paper.

Permanent link to this version:

http://urn.kb.se/resolve?urn=urn:nbn:se:bth-18989 


\section{Framework for Analysis of Multi-Party Collaboration}

\author{
Yuliyan V. Maksimov'13 \\ Institute for Interactive Technologies \\ FHNW University of Applied Sciences and Arts \\ Northwestern Switzerland \\ Windisch, Switzerland \\ \{yuliyan.maksimov, samuel.fricker\}@fhnw.ch
}

\author{
Samuel A. Fricker ${ }^{12}$ \\ Software Engineering Research Laboratory (SERL-Sweden) \\ Department of Computer Science and Engineering (DIDD) \\ Blekinge Institute of Technology \\ Karlskrona, Sweden \\ \{yuliyan.maksimov, samuel.fricker\}@bth.se
}

\begin{abstract}
In recent years, platforms have become important for allowing ecosystems to emerge that allow users to collaborate and create unprecedented forms of innovation. For the platform provider, the ecosystem represents a massive business opportunity if the platform succeeds to make the collaborations among the users value-creating and to facilitate trust. While the requirements flow for evolving existing ecosystems is understood, it is unclear how to analyse an ecosystem that is to be. In this paper, we draw on recent work on collaboration modelling in requirements engineering and propose an integrated framework for the analysis of multi-party collaboration that is to be supported by a platform. Drawing on a real-world case, we describe how the framework is applied and the results that have been obtained with it. The results indicate that the framework was useful to understand the ecosystem context for a planned platform in the domain of artificial intelligence, allowed identification of platform requirements and offered a basis to plan validation.
\end{abstract}

Index Terms-platform and ecosystem requirements, collaboration modelling.

\section{INTRODUCTION}

To benefit from unprecedented levels of innovation, many businesses see a need to look beyond their own walls for new ideas and collaborate with partners to develop solutions that solve relevant challenges [1]. For example, a group of collaborators won a global competition for accurately predicting prostate cancer, even though they had not been active in cancer research. The competition was hosted and managed on a cloudbased platform that facilitated the collaboration. The platform has helped level the competitive playing field by making it easy to connect with others whose expertise and skills were needed for solving the challenge and giving access to hardware, software, and tools needed to develop the idea.

Platforms enable an unprecedented level of value creation by creating ecosystems of potential collaborators [2]. An ecosystem consists of a platform owner, providers responsible for the interface to the users, e.g., mobile device producers are providers for the Android platform, and platform content producers and consumers as users of the platform [2]. Platforms allow companies to open their in-house value chains and connect providers that may offer useful inputs with consumers that benefit from the output of the shared value chain. An example of such a platform is the Apple App Store, a marketplace that connects app developers and smartphone owners. A B2B example is Bonseyes (Bonseyes.eu), a marketplace for artificial intelligence (AI) that connects data providers, data scientists, and systems engineers for joint development of AI systems [3]. Moving from traditional software product development to a platform business requires a deep understanding of the collaboration between the external producers and consumers that eventually will be implemented in the platform and persuasion of these parties to join the ecosystem and be active members.

Substantial research has been performed for understanding how to manage and evolve a platform and its ecosystem. Jansen et al. have spawned a whole community to research software ecosystems intending to enable their management [4]. Fotrousi et al. proposed to manage ecosystems by monitoring key performance indicators (KPIs) [5], and Jansen to use the KPIs for managing ecosystem health [6]. To facilitate the evolution of ecosystems, Knauss et al. proposed a process and method for continuous elicitation and clarification of requirements [7].

Little is understood, however, how a so far inexistent ecosystem should be built [8]. This challenge is experienced by the Bonseyes marketplace that seeks to create an ecosystem of industrial corporates that want to collaborate with data scientists for jointly developing systems of artificial intelligence. We focus on the analysis of the collaboration in a future ecosystem and offer a complementary method to elicit the requirements for a platform intended to support it.

Our paper assumes that the platform may be built by implementing requirements identified by analysing cases of multi-party collaborations that ought to be supported by the platform and propose a framework for such analysis. Rather than creating yet another way of modelling, we combined techniques that have been researched in the requirements engineering (RE) field and extended the combination with methodical guidance.

Our paper gives an overview of methods explored in the last 15 years by the requirements engineering community for analysing multi-party collaboration. These methods were composed into a proposed integrated framework for semi-formal modelling of multi-party collaboration. For early validation, the framework was applied on a case for multi-party collaboration typical for the Bonseyes platform: Kaggle-like competitions [9]. These are competitions where participants create specific data analysis algorithms to solve predefined AI challenges. We describe the obtained modelling results together with the methodological tactics that surfaced requirements that were relevant for platform design and ecosystem community building. 
Section 2 describes existing research in RE for the analysis of multi-party collaboration. Section 3 describes our proposed framework, and Section 4 the approach chosen for initial validation of the framework. In Section 5, we present our results and in Section 6, the analysis of the results. Section 7 discusses the contribution, and Section 8 concludes.

\section{ANALYSIS OF MUlti-PARTy COLLABORATION}

In the following, we first look into the definition of the term collaboration and derive constructs relevant to its characterisation. Afterwards, we take a closer look at the representation of collaboration in the field of RE by introducing our literature study on collaboration modelling techniques in the RE literature. Finally, we also discuss tactics found in the literature, which the authors utilise to more precisely describe their approaches and enhance their analysis.

\section{A. Collaboration Constructs}

In an article on online collaboration in the context of the Architecture, Engineering and Construction industry Ilich et al. [10] cite the work of Hobbs [11] and define collaboration as "the agreement among specialists to focus their abilities in a particular process to achieve the longer objectives of the project as a whole, as defined by a client". In the context of virtual organizations (VO), which are independent companies who share competence and resources through the integration of information system infrastructure [12], Priego-Roche et al. define collaboration as "compromises to integrate competences and resources" [13].

As there are many concepts mentioned in these definitions similar by meaning in the following, we synthesise them and define our constructs of collaboration (bold terms). First, an agreement can be seen as a compromise, and it is the more general term that expresses the recorded rules for working together as well as for creating and exchanging resources and knowledge. Specialists and clients can be independent companies, organisations or individuals and are the actors or the parties in the collaboration. We use the terms interchangeably. Since we have multiple actors in the collaboration, we speak of a multi-party collaboration. The abilities or the competences are the skills utilised by actors to achieve the goals of the collaboration. The objectives are the goals of the collaboration. The resources are material or immaterial goods like hardware or software utilised to achieve these goals. The collaboration process is a sequence of activities where competences and resources are integrated to achieve the goals of the collaboration.

\section{B. Literature Analysis}

We examined the literature on multi-party collaboration in the field of RE and state of the art on commonly used techniques to model the collaboration. We asked the question: How is the collaboration between stakeholders of a system modelled in the $R E$ literature? We targeted the journal of Requirements Engineering and studied all the titles and abstracts of the articles published in the last 15 years, which is the period between March 2005 and March 2019. As Kitchenham \& Charters recommend for systematic literature reviews [14], although we did not perform one, we defined a criterion to select in scope papers: the title and abstract had to indicate collaboration between stakeholders in any sense. Collaboration between system developers and stakeholders of the system was let out of scope since we focus on the interactions between actors to collaborate and not on interactions between developers and stakeholders to design a system. Although we did not define explicit exclude criteria, we had a rationale for every decision and documented all. We identified 24 articles being in scope. After a closer examination of the full-text articles, we were able to discard additional 10 leaving us with a set of 14 articles to work with and analyse the modelling of the interactions between the stakeholders of a system. We identified used notations. If they were new, we extracted constructs from the proposed language. If the notations were known, e.g., $i^{*}$ we noted the language down and identified the literature that defines the language. Mainly the first author was involved in the selection of the papers while the second author was reviewing the decisions and resolving doubts.

All in all, the published works differ substantially in the units considered relevant and the modelling techniques used for analysis. Table I gives an overview of these units and techniques.

Several authors considered the definition of the ecosystem in which collaboration takes place to be important. According to these views, an ecosystem consists of actors that have relationships with each other. Buchmann \& Karagiannis [15] describe companies, their departments, and employee roles that participate in collaborative business processes. Alreshidi et al. [16], and Garde \& Knaup [17] describe data exchange pathways to characterise possible communication channels in a network of collaborating roles or organisational units. Ballejos \& Montagna [18] put these inter-organisational networks into a broader interorganisational context by separating the members and nonmembers of a network.

Two works extended the ecosystem analysis with a specification of forces that are exerted among the actors. Drawing on Porter's Five Forces framework [19], Pijpers et al. [20] specify forces of various strengths among actors in the ecosystem. Priego-Roche et al. [13] extend this analysis with documentation of grouping, regulation, complementing, and competition relationships among the actors.

Many authors consider the intentions of the actors identified in the ecosystem to be of central importance for the analysis of their collaboration. Knauss et al. [7] consider business models of these actors to be an important source of strategic requirements for ecosystem evolution. Daneva \& Wieringa [21] formalise this by mapping how information sharing among the collaborators interacts with the actors' benefits, cost, and resistances.

Well represented is the $i^{*}$-inspired [22] goal modelling family of analytical frameworks for modelling intentions and how these intentions are refined or delegated among the actors. Gharib et al. [23], Dalpiaz et al. [24], and Bryl et al. [25] use the Tropos framework [26], Barata \& da Cunha [27] use the user requirements notation URN [28], and Patricio et al. [29] the nonfunctional requirements NFR framework [30]. The research works differ in the relation of the goal to other concepts: information and information quality [23], goal monitoring [24], social networks [25], and collaboration process [27], [29]. Even though outside the $i^{*}$ modelling family, also Knauss et al. [7] 
and Priego-Roche et al. [13] consider goals to be essential sources for requirements governing the ecosystem evolution, respectively the collaboration processes.

Some works have modelled the value chains that emerge when actors pursue their intentions in the form of networks of value exchanges. Knauss et al. [7] model software supply networks SSNs [31]. Pijpers et al. [20] extend the networks with specifications of value exchanges based on $e^{3}$ value models [32]. Priego-Roche et al. [13] follow the actor dependencies ideas present in the $i^{*}$ family of models and specify availability, investment, personnel, function, and regulation commitments between the actors in the ecosystem.

Most authors translated the so far specified ecosystem structures into operational business processes that describe the series of activities performed by the actors to fulfil joint or individual intentions. Pijpers et al. [20] and Barata \& da Cunha [27] use activity diagrams, and Priego-Roche et al. [13] and Wolter \& Meinel [33] BPMN models, both widely established modelling notations. Buchmann \& Karagiannis [15] used their own business process modelling notation that resembled activity diagrams extended with cross-diagram links. Patricio et al. [29] modified the activity diagrams to describe blueprints of service experiences. Gharib et al. [23] proposed and used WFA-nets to describe the workflow among actors and analyse the propagation of information quality. Buchman \& Karagiannis [15] added state charts to describe the orchestration performed by the platform to coordinate the collaboration among the actors within the previously specified business processes.

The last unit used to analyse collaboration was information provided, shared, and used in the multi-party collaborations.

TABLE I. MOdELling OF MULTi-PARTy COLLABORATION (*: NO PARTICULAR NOTATION PROPOSED OR USED)

\begin{tabular}{|c|c|c|}
\hline $\begin{array}{l}\text { Unit of } \\
\text { Analysis }\end{array}$ & $\begin{array}{l}\text { Central } \\
\text { Aspect }\end{array}$ & Modelling Technique \\
\hline \multirow[t]{2}{*}{ Ecosystem } & $\begin{array}{l}\text { Network of } \\
\text { Actors }\end{array}$ & $\begin{array}{l}\text { Business Entities [15] } \\
\text { Stakeholder Network [16] [17] } \\
\text { Inter-organisational Context [18] }\end{array}$ \\
\hline & Forces & $\begin{array}{l}\text { e3 forces [20] } \\
\text { Virtual Organisation Alliances [13] }\end{array}$ \\
\hline \multirow[t]{2}{*}{ Intentions } & $\begin{array}{l}\text { Business } \\
\text { Rationales }\end{array}$ & $\begin{array}{l}\text { Business Model* [7] } \\
\text { Problem Dependency Map [21] }\end{array}$ \\
\hline & $\begin{array}{l}\text { Actor } \\
\text { Goals and } \\
\text { Dependenci } \\
\text { es }\end{array}$ & $\begin{array}{l}\text { Tropos [23] [24] [25] } \\
\text { User Requirements Notation URN [27] } \\
\text { Non-functional Requirements (NFR) } \\
\text { Framework [29] } \\
\text { Business Goals* [7] } \\
\text { Common Objectives [13] }\end{array}$ \\
\hline Value Chain & $\begin{array}{l}\text { Value } \\
\text { Exchanges }\end{array}$ & $\begin{array}{l}\text { SSN [7] } \\
\text { e3 value [20] } \\
\text { Collaboration Willingness [13] }\end{array}$ \\
\hline \multirow[t]{2}{*}{$\begin{array}{l}\text { Business } \\
\text { Process }\end{array}$} & Workflow & $\begin{array}{l}\text { Activity Diagram [20] [27] } \\
\text { BPMN Models [13] [33] } \\
\text { Business Process [15] } \\
\text { SEB Diagrams [29] } \\
\text { WFA-nets [23] }\end{array}$ \\
\hline & $\begin{array}{l}\text { Orchestrati } \\
\text { on }\end{array}$ & State Charts [15] \\
\hline \multirow[t]{2}{*}{ Information } & $\begin{array}{l}\text { Information } \\
\text { Model }\end{array}$ & $\begin{array}{l}\text { ER Diagram [15] } \\
\text { Class Diagram [17] } \\
\text { Building Information Model* }{ }^{*}[16]\end{array}$ \\
\hline & $\begin{array}{l}\text { Information } \\
\text { Quality }\end{array}$ & Information Quality Dependencies [23] \\
\hline
\end{tabular}

Buchman \& Karagiannis [15] and Garde \& Knaup [17] use established diagrams to model that information: entityrelationship diagrams and class diagrams, respectively. Alreshidi et al. [16] referred to a building information model $\mathrm{BIM}$ as a repository of information shared among collaborating actors in the construction domain.

Gharib et al. [23] modelled the propagation and use of the information in the multi-party business processes and analysed information quality dependencies.

While no single research had addressed all units of analysis, the works indicate that five units of analysis should be described for analysing multi-party collaboration in platform-supported ecosystems. These units are the actors and forces making up the ecosystem, the business rationales and goals reflecting the intentions of the actors, the value exchanges that characterise the value chains, the workflow and orchestration characterising the business processes, and the definition of data and quality characterising information shared among the actors. Each unit of analysis was addressed by at least three independently performed research works. These repeated considerations give the confidence that the units of analysis are of relevance for requirements engineering and not just an artefact of the specific problem or domain that was addressed by single research.

\section{Analysis Tactics}

In these studies, we were also searching for tactics utilised by the authors to increase the clarity in the description of their approaches and as a consequence to enhance their analyses. We use these tactics in our framework, discussed in Section III, to analyse and gain a deeper understanding of the observed case of collaboration. Table II summarises the findings.

Buchmann \& Karagiannis [15] shift between abstract and concrete notations of interaction elements of a system and are so able to support stakeholder decision making. The authors of [7], [16], [20] and [27] all discuss problems, challenges, barriers, and issues to understand the concerns of stakeholders in the particular study better. Priego-Roche et al. [13] use a goals refinement tactic to break bigger stakeholder goals into more concrete and operationalisable goals. Dalpiaz et al. [24] refine goals and implement a goal monitoring system in order to enhance the governance of their fulfilment. Ballejos \& Montagna [18] use checklists to characterise the roles of stakeholders. Knauss et al. [7] elicit information about practices applied by an actor in the ecosystem to better understand the requirements analysis process of a development team and its interactions with other actors. Gharib et al. [23] mathematically define reachability of configurations of WFA-nets and propose a graphical representation of the reachability of all possible configurations in such nets.

TABLE II. ANALYSIS TACTICS

\begin{tabular}{|l|l|}
\hline \multicolumn{1}{|c|}{ Tactic } & \multicolumn{1}{c|}{ Utilised in } \\
\hline Shifting between abstract and concrete & {$[15]$} \\
\hline Elicitation of Problems, Challenges, Barriers, Issues & {$[7],[16],[20][27]$} \\
\hline Goal refinement & {$[13],[24]$} \\
\hline Checklists & {$[18]$} \\
\hline Elicitation of Practices & {$[7]$} \\
\hline Reachability & {$[23]$} \\
\hline
\end{tabular}




\section{ANALYSIS FRAMEWORK}

While we focus on multi-party collaboration characteristics, in the literature, there is a variety of frameworks that focus on Business-IT alignment. The architecture of these frameworks, combined with the literature findings on collaboration described in Section II, create the foundation of our Multi-Party Collaboration Analysis Framework (MP-CAF). In the following, we first discuss existing and then our framework.

\section{A. Inter-Organisational Business-IT Alignment Frameworks}

There are frameworks like the business process management framework ARIS [34] and the enterprise architecture frameworks like The Zachman Framework [35] or TOGAF [36] that focus on intra-organisational Business-IT alignment. Our multi-party approach is on an inter-organisational level, and we discuss frameworks that describe inter-organisational Business and IT perspective alignment more in detail.

While Priego-Roche et al. [13] for example, differentiate between three organisational levels in their framework for $\mathrm{VO}$ requirements elicitation: intra-, inter- and extra-organisational. Pijpers et al. [20] based on Derzsi \& Gordijn [37] write about intra- and inter-organisational alignment between perspectives in networked value constellations [38]. These constellations are organisations working together to satisfy a complex customer need [37]. Our definition of collaboration networks (CNs) is similar but brother since we allow not only organisations but also individuals to collaborate to achieve a common goal.

Pijpers et al. further introduce an inter-organisational business-ICT alignment framework called $e^{3}$ alignment, which has four perspectives [20]: "Business Strategy", "Value Creation", "Processes" and "IT/IS". These are introduced before in a similar way in the work of Derzsi \& Gordijn [37].

\section{B. Multi-Party Collaboration Analysis Framework (MP-CAF)}

Our conceptual framework, which "is a set of definitions of concepts" [39], for the analysis of multi-party collaboration is illustrated in Fig. 1. Before the description of the framework, we provide some definitions.

The collaboration between the parties of an ecosystem we also call ecosystem collaboration (EC). The parties are actors in ecosystem collaboration processes (ECPs), which denote the processes to achieve the common goals of the collaboration network $(\mathrm{CN})$. A collaboration network is formed by the actors in the ECPs and their relationships. An actor can be an individuum, group of individuals, organisation or even group of organisations that have unified goals and appears as one entity to the CN. Systems that collaborate without human intervention are resources belonging to one or more of these actors.

We aim to analyse and characterise the EC. For that, we reflect on the units of analysis shown in Table I and consider the goals, value and processes perspectives of the discussed alignment frameworks. Based on that, we propose the following EC characteristics: Context, Goal, Value, Process, Information.

In terms of our framework, we speak of EC characterisation levels $(\mathrm{ChL})$ that build the set of definitions for our framework:
- Context - At this level, the $\mathrm{CN}$ actors and the relationships between them are identified. Here also the actors outside the network influencing or having relations to the $\mathrm{CN}$ members and affecting the $\mathrm{EC}$ are identified. The actors are chosen based on their competences, and they can be projected into their entity names, e.g., naming a stakeholder "Artificial Intelligence Freelancer" suggest that the actor has data science skills.

- Goal - At this level, the intentions in terms of the goals of the $\mathrm{CN}$ members are identified, and the required agreements are initially discussed. Their content is refined through discussions on the next levels.

- Value - Here, the values, which are resources interchanged between $\mathrm{CN}$ members to achieve the $\mathrm{EC}$ goals, are more precisely discussed.

- Process - This is the level where the ECPs needed to achieve the EC goals are characterised and described.

- Information - This level is about specifying the concrete type of information and its properties flowing between the actors to achieve the goals of the EC.

Fig. 1 illustrates these levels. For each level, based on the literature, we suggest a number of different well-known notations. There are also some exceptions, like on the Context level, where we find some of the custom notations observed in the literature to be very helpful. In general, our framework has a descriptive and not a prescriptive nature. As seen in Table I, there are also additional and custom notations that authors utilised. It is up to the experience and preferences of the people utilising the framework to use the one or the other notation.

Fig. 1 also demonstrates the process nature of the EC characterisation. The process of characterisation goes in two directions: from being more abstract to becoming more concrete, and iterating between the ChLs, which means to redefine something that has been defined before. These directions are also the place where two of the identified tactics in Table II come into play. One can iterate between the levels by seeking to identify Problems, Challenges, Barriers and Issues on each of them and think of how they might affect the others. On each level, one can start an abstract discussion and try to get as concrete as possible.

Concerning the rest of the tactics, the goal refinement tactic, for example, is about asking questions that increase the granularity of the goals, and it is especially suitable for the Goal level. Checklists can be utilised specifically on the Context level, for example, to classify the stakeholders of the collaboration. Elicitation of practices about how something is done and what information is needed can be valuable for the Goal, Value, Process and Information levels in particular. Defining reachability is something of particular interest for the WFA-nets.

Although we recommend following the order of the levels to characterise the EC, it finally depends on the aims and context of the specific case where the framework is intended to be utilised. For example, the information and process characteristic of EC can be defined interchangeably. It might be easier to define the types of information that need to be exchanged and their properties after it is clear what are the steps in a process, but depending on the case also the opposite might be valid. With 
other words, that first, the information needed for a process to happen, needs to be defined. In most cases, there will be a need to jump between levels to get more concrete in the characterisation of the EC.

Our framework aims to describe the collaboration from different perspectives, which is achieved through the diagrams defined on the different ChLs. Primarily it is an instrument for requirements engineers or others having the qualification to work with the enumerated diagram types and the goal to elicit requirements for a planned platform aimed to support the collaboration between its users. These users and the engineers are, thus, stakeholders of the framework. The engineers are the ones who choose the notation for each $\mathrm{ChL}$, and they choose the tactics to elicit requirements while discussing the models.

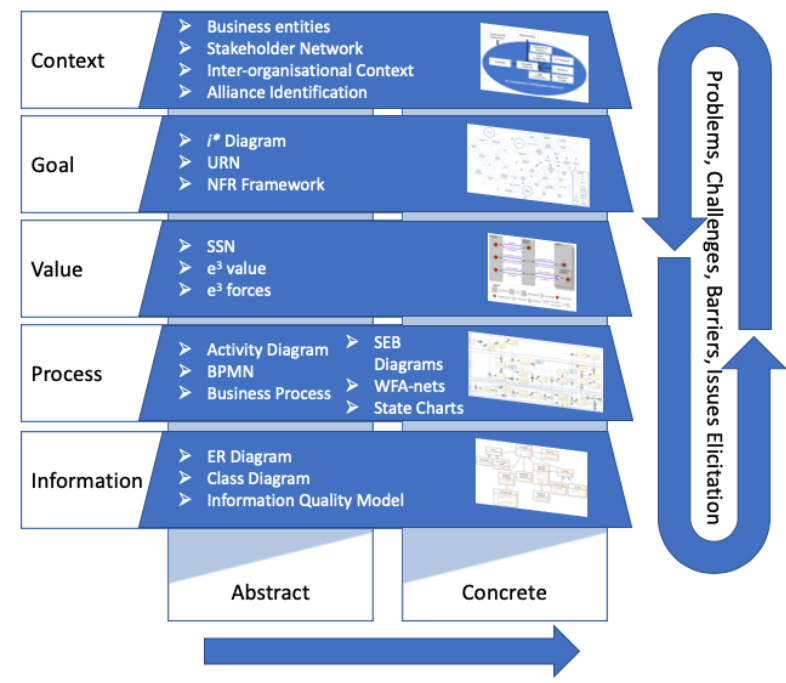

Fig. 1. Multi-Party Collaboration Analysis Framework (MP-CAF).

\section{VALIDATION METHOD}

For the validation of the proposed framework, we are generally guided and use the technology transfer model of Gorschek et al. [40]. They present a way to transfer research findings in industry practice. Based on our experience in large consortia in European projects, where various organisations collaborate, we find the model generalizable enough to be applied in areas of practice other than industry. For example, medical institutes like the case reported in the research at hand.

The model consists of seven steps: 1) identify the issue, 2) formulate the problem by studying state of the art, 3) design a candidate solution, 4) validate in academia for example through lab experiments, 5) perform a static validation in practice, for instance through interviews, 6) perform a dynamic validation, for example through piloting and 7) release the solution. The authors emphasise the need to adjust the model to the particular case, where it is applied. In our study, we adjust the model by not performing an academic validation since we have a chance to directly validate in practice and apply our framework in a realworld case. However, additional validation is needed before we pursue with steps 6 and 7. Here we report on steps 1, 2, 3 and 5 .

We designed our framework to have an instrument for a better understanding of the collaboration between the actors in a
$\mathrm{CN}$ and for requirements elicitation of the platform supporting the EC. For validating the framework, we ask VQ1: Do the users of the analysis framework consider it sufficient to describe the discussed collaboration? Sufficient we define as complete in terms of describing all the identified collaboration constructs defined in Section II A: agreement, actors or parties, competences, goals, resources and collaboration process.

We want our framework to be broadly utilised, and besides asking for sufficiency, we are also interested in how are the modelling notations perceived and where are the challenges of their application. Thus, we ask VQ2: Do the users of the analysis framework consider the chosen modelling notations to describe the discussed collaboration useful? Useful we define as a notation being understandable, easy to use to describe a particular collaboration construct, and supportive to elicit requirements for a collaboration platform. In that sense, the two questions are complementary to each other; while VQ1 asks about a complete description of the collaboration, VQ2 is about having the right instruments to obtain it.

We aim to gain an in-depth understanding of the collaboration characteristics in a $\mathrm{CN}$ and thus utilise the suggested framework in a semi-structured expert interview to elicit the requirements for a planned platform aiming to support the EC. We apply the recommendations for preparing, conducting and reporting such an interview in the field of Software Engineering (SE) summed from literature and the experience of Hove \& Anda in [41].

The chosen real-world case [42] is in the context of increased utilisation of surgery rooms. Maintaining surgery rooms and their equipment is one of the main cost factors for hospitals. An AI competition is to be organised to develop AI object detection models (AI models) that detect instruments, organs and pieces of equipment like bags, clips and threads by analysing a video feed during a surgery. These AI models are a part of a larger AI application that aims to follow the progress of a surgery so that the next patient is prepared on time for the following surgery.

The case was mainly selected because there is a planned ecosystem consisting of the platform and its stakeholders. The platform would support the AI competition. The development of the platform is done in the context of the previously mentioned EU Horizon 2020 project "Bonseyes". The main stakeholders of the platform for this particular case are the competition initiator (CI), the competition organiser (CO) and the competition participants (CPs). The second author is mainly responsible for the RE performed in the project.

We utilised our framework to understand the collaboration between the stakeholders better and to elicit requirements for the planned platform. Both authors were the requirements engineers utilising the framework and the interviewee, who is a researcher in a clinical institute in Germany in the field of applied AI, was the representative of the feature CI organisation. Thus, the three of us were the users of the analysis framework. We did not have explicit representatives of the two other stakeholder groups of the platform: $\mathrm{CO}$ and CPs. However, the first author is following Kaggle competitions in the role of a participant and has experience in organising Hackathons. The second author has organised different types of Hackathons, also such utilising AI 
technologies. Finally, he as well has the main responsibility for the RE in the project where the planned platform is developed. The plan for the future is the platform to take the role of the CO.

To discuss the Context ChL, we choose a diagram similar to the one presented in [13] (see Fig. 2). For the Goal ChL, we choose the commonly used $i^{*}$ diagram [22] (see Fig. 3). For the Value ChL, we choose a simple $e^{3}$ value model [20], [32]. For the Process ChL, we utilised the commonly used BPMN diagram [43]. And for the Information ChL, we developed a class diagram [44].

The interview took place online using the online platform GoToMeeting (https://www.gotomeeting.com/) and had three parts on different days in the first week of April 2019. The participants were the interviewee and the two authors in the first two parts and the first author and the interviewee in the third part. We prepared an interview guideline with the models and the questions for the interview and sent them to the researcher two days before the interview. The first part covered the Context and the $i^{*}$ models lasting for approx. $60 \mathrm{~min}$. We received feedback and changed the models for the second part where we discussed the changes, the next two models $e^{3}$ value and BPMN, and the rest of the questions of the interview guideline. The second part lasted for approx. $100 \mathrm{~min}$. The third part was about the Information model and overall question about the platform requirements and continued for approx. $20 \mathrm{~min}$. The interview was in the native language of the interviewee German. We recorded the video stream from the cameras, the audio stream and the shared screen in the sessions.

\section{V.RESULTS}

To demonstrate the application of our Analysis Framework, we present our models for the first two ChLs and highlight some of the discussions which led to their change. We note that the elicited requirements at this stage are to be treated as "wishes" of one of the stakeholders of the platform and need to be further discussed with the rest of the ecosystem partners.

\section{A. Context}

The left side of Fig. 2 shows our initial model for the context of collaboration in the case under investigation. The organisations in the blue ellipse are the members of the $\mathrm{CN}$. Outside the ellipse are organisations that are extern to the $\mathrm{CN}$, however with interest to it. The $\mathrm{CI}$ and the $\mathrm{CP}$ are communicating only through the $\mathrm{CO}$. There are reporters and viewers interested in the network and recruiters such as headhunters interested in the participants but again only communicating with them through the $\mathrm{CO}$.

During the interview, we concretised the model by discussing which role would be taken by whom exactly (realworld actors) in the EC. We defined the roles as shown on the right side of Fig. 2; e.g., CPs can be: small or large companies, AI freelancers, students, research groups or networks of partners. We also had a number of additional discussion points and changes. We comment on two of them, which highlight, first the limitations of this diagram type, and then its usefulness for the improved understanding in the case:
Communication arrow between CI and CP - The interviewee suggested that he as part of the future CI organisation might want to get in touch with the CPs after the competition. However, this was a time-based request "after the competition" which our model was yet not able to express. Our model at the Context characteristic level was designed based on others observed in the literature, but the request showed its limitations and the need for future work.

Different interpretations of the role "Recruiter" - The first author designed the model by having a "Head Hunter", who generally searches for talented data scientists in mind, for the actor "Recruiter." During the discussion, it became clear that the interviewee and the second author had different understandings of the term: a person who recruits participants for the competition, or a person who recruits staff for the $\mathrm{CI}$ or $\mathrm{CO}$.

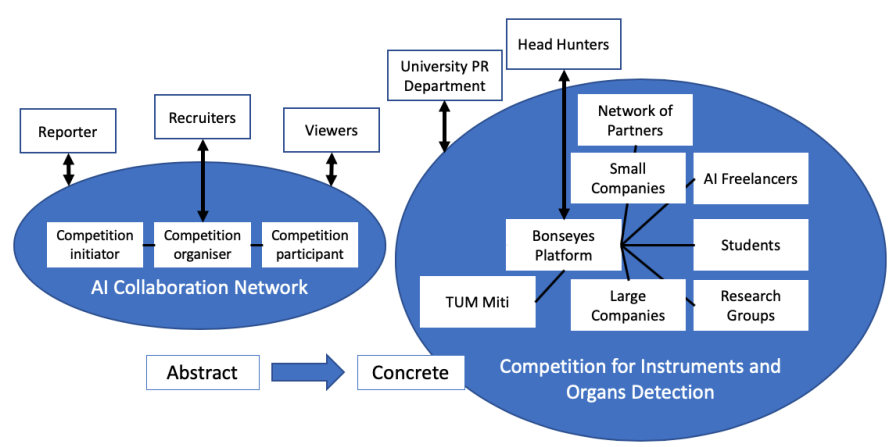

Fig. 2. Initial Context model proposed for the AI competition collaboration network before the interview (on the left) and updated Context model after the interview (on the right).

We were also able to elicit requirements for the planned collaboration supporting platform. Here some examples: Defining CPs - A participant in the competition could be a person, a group of people, an organisation or a group of organisations. Roles of stakeholders - CN members like a person, an organisation, or a group can have internal or external actor roles in the $\mathrm{CN}$ depending on the context, e.g., a researcher can be the CI and also the "Reporter" of the competition by writing a scientific publication and reporting data in it, collected during the competition.

\section{B. Goal}

The left part of Fig. 3 demonstrates a part of our initial goal model for the observed CN. A CI has the task to organise a competition. The organisation needs to be quick and cost low effort. The outcome should be of having high-quality AI modes. Letting a $\mathrm{CO}$ organise the competition means less effort and faster organisation of the competition for the CI. However, he still needs to provide the prize money and evaluate the AI models provided by the CPs through the $\mathrm{CO}$. Evaluating the AI models, however, slows the process of organising a competition for the CI.

During the discussion of the initial $i^{*}$ model, we again tried to obtain concrete information from the $\mathrm{CI}$ and understand if we correctly assumed the goals, tasks and the resources being exchanged. We highlight an example of the discussed topics 
here that led to changes in the model; the affected part of the resulting model is shown on the right side of Fig. 3:

Discussion of Alternatives. In our initial model, there were no alternatives to the organisation of a competition. At this stage, though, alternatives could be discussed, e.g., development of AI models by students, in addition to organising a competition. Both approaches have advantages and shortcomings to achieve the overall goals of the CI, e.g., an advantage in the case of development with students is that the dataset must not be made public, and an advantage of the competition is that "the knowledge of the mass" is utilised, as the interviewee noted.

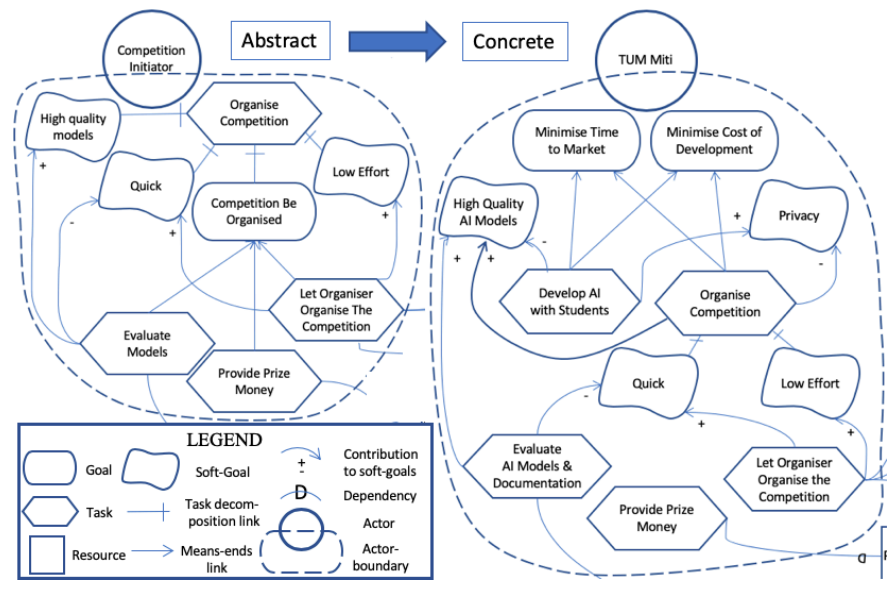

Fig. 3. Part of the initial $i^{*}$ model proposed for the AI competition collaboration network before the interview (on the left) and part of the updated model after the interview (on the right).

We also had discussions that helped elicit requirements for the planned platform. We exemplify one of them:

Return on investment (RoI) for the CI. The CI in the concrete case would be a medical research institute. It would provide an annotated dataset to the competition. However, by doing so, it also would make the dataset public and risk losing ownership of it. The questions for the platform in planning would be, how to protect the intellectual property (IP) of the CI.

Similarly, as described for the Context and $i^{*}$ diagrams, we discussed the $e^{3}$ value diagram, the BPMN process diagram and the Information class diagram.

\section{VI.ANALYSIS}

In the following, we answer our two validation questions and present threats to validity as well as our plans for future research on how to mitigate them.

\section{A. Answers to the validation questions}

In Section II A we extracted collaboration constructs from the definition of the collaboration concept in the literature. To answer the question about the sufficiency of the framework to express these constructs, we state what is defined where. We defined the actors specifically on the Context level through the Context diagram. We also implicitly defined their competences by giving them appropriate role names like Head Hunters and AI Freelancers. At the Goal level, we defined the goals of the collaboration through an $i^{*}$ diagram. The Value level and the $e^{3}$ value diagram helped us specifically to focus on the exchanged resources. In the same manner, we utilised the Process level and the BPMN notation to express the process of collaboration. Agreements passed between $\mathrm{CI}, \mathrm{CO}$ and CPs were visible in the $i^{*}, e^{3}$ value and BPMN diagrams. We also asked our interviewee if he misses something in the description of the collaboration, which he denied. Thus, for the given case, we conclude that the framework was sufficient to describe the collaboration.

Regarding the second question, if the notations in the framework were useful, we made the following observations: While the Context diagram can express some of the participants' competences implicitly, it still might not be able to express all of them since an actor as "Students" is not expressive enough, and an actor name like Recruiters can cause misunderstandings. The $i^{*}$ model was not easy to use. There were too many new concepts for the interviewee. He needed time to understand them and follow the trace in the diagram to perceive the relations. The $e^{3}$ value diagram was simple enough to be understood, although it was also new for the interviewee. He was already familiar with and actively using BPMN and class diagrams and thus the understandability there was given. However, we draw a detailed BPMN diagram and thus had to make many decisions, and the participant stated: "One can ask himself if many assumptions are always valid, or if they are inviolable [...]".

We experienced that each of the models has its strengths and weaknesses, and combining them provides a better view on and understanding of the ECP in the observed case as for applying each of the models stand alone. Thus, we conclude that although some of the diagrams are not easy to process their usefulness is improved and given partially because of the framework.

\section{A. Threats to Validity}

The presented validation of the proposed analysis framework is only preliminary. It describes the use of the framework, shows results that can be achieved with it, and reports how the value of the results may be enhanced with suitable analysis tactics. Following the taxonomy of threats to validity proposed by Runeson [45], there are still substantial threats to internal and external validity. The presented early validation showed that the framework leads to a definition of multi-party collaboration approach that meets the goals of the interviewee. However, it did not show the use of the framework to cover additional cases of multi-party collaboration and not demonstrate yet that it would lead to an ecosystem that is attractive to be joined and produces the outcomes that the platform developer hopes for. Also, it did not demonstrate models would enable the development of a successful platform or suffice for the definition of scenarios and criteria for validation and evaluation of the platform. Finally, even though the proposed framework is composed of earlier partial approaches to collaboration analysis, the paper did not demonstrate that the framework applies to other platforms and ecosystems. Such extended validation is, however, in planning.

The paper describes the designed artefact, the analysis framework and the results of a static validation in practice through interviews with one future user of the collaboration platform. The validation is step 5 in the model of Gorschek et al. [40] about technology transfer in practice. This early 
investigation is about the application of the artefact on the case of Kaggle-like competitions. We have recorded the interviews and stored them in our research repository together with the evolving models. Also, we revisited the recordings to ensure a chain of evidence between the presented results and the raw data collected in the study. We believe that threats to construct validity are small as the models we have presented match those suggested by the underlying literature. Still, there are important reliability threats present as we do not know yet to what extent the results are stable when the study would be replicated. Such replications are also subject to planned future research.

\section{VII.DISCUSSION}

In this paper, we have proposed a framework for analysing multi-party collaborations. The framework is intended to be used by developers of platforms that shall support so far inexistent ecosystems of platform users. The framework offers a tool that complements earlier approaches for platform requirements engineering, such as the negotiation-focused process of Intel that assumes great expertise of platform use by the negotiating stakeholders [46] or the requirements-flow approach that is useful when the ecosystem already exists, and the platform should be evolved [7]. We have shown how the framework is intended to be used for the analysis of one scenario of multiparty collaboration: collaboration in Kaggle-like competitions for the development of AI systems. The platform developer intends to continue using the analysis method for analysing further cases of multi-party collaboration that are to be supported by the platform, beyond the format of competitions, to achieve a comprehensive understanding of ecosystem context and requirements for platform development and validation.

The analysis framework, shown in Fig. 1, is a synthesis of previously proposed approaches for modelling multi-party collaboration. It is more complete as a framework as it is the first one that covers all five units of analysis that were used before. The framework was instantiated in the presented case by modelling of inter-organisational Context [18] to describe the ecosystem, $i^{*}[22]$ to reason about actor intentions, $e^{3}$ value [32] to specify the value exchanges between the involved actors, BPMN to describe the business process to be used by the actors for the value exchanges, and a class diagram to provide an overview of the information used and assets exchanged between the actors. The choice of the diagrams was based on the richness and precision of the specifications that they allowed. Each diagram provided a unique perspective of the multi-party collaboration by describing at least one aspect that was not visible in any other diagram, except the class diagram. Instead, the class diagram summarised the information and assets used and exchanged in the value chain and business process views. All diagrams were considered relevant by the interviewee, and no need for further units of analysis or diagrams to describe the collaboration emerged during the interviews.

We are aware, however, that for the full set of requirements to build a platform, our framework will need to be combined with additional "classical" elicitation techniques like GUI prototypes, or such that are favourable due to the context of the observed case like state machines.
We are also aware that although security and privacy concerns were not discussed with the interviewee, NFRs like these are an essential topic that needs to be considered in our framework. NFRs express qualities of the platform to be and are often used to decide about alternative solutions of an issue. A goal-oriented modelling method, like the GRL [47], can be used to evaluate different alternatives [48]. Specifically, soft goals can be used to justify alternatives and refine goals, and they are the ones that usually reflect qualities. Thus, these concerns can be handled on the Goal level in our framework.

Regarding the utilised diagrams, some of them were difficult to use. The goal models required in-depth discussions to understand their meaning, and the process diagram was cluttered with decisions that appeared to be too detailed and premature. Still, the early validation presented in this paper indicated that the framework components were relevant and the overall framework complete from the perspective of a potential actor in the future ecosystem.

An open question that we will be further researching, however, is if the full potential of the notations in terms of using all the constructs in a language is needed to describe the collaboration between parties. For example, we only used the basic version of the $i^{*}$ notation [22], and we perceived it as sufficient to describe the particular case of an AI competition. Nevertheless, we also see advantages of increasing the expressiveness of the language, e.g., by adding decision supporting AND/OR goal decomposition constructs [26] for describing if the AI models are to be developed with students or through a competition. We recommend starting with as few constructs as possible for a notation, which, however, are sufficient to describe the collaboration constructs described in Section II A. This would minimise the cognitive load for users of the framework not familiar with the type of utilised notation.

A cost-effectiveness analysis is as well a topic of future work. Every ChL contributes an additional view on the ECPs. However, it also causes an increased complexity of the collaboration description and increases its cost in terms of the effort needed to create and maintain the additional model.

During the application of the models, needs emerged to draw conclusions about the multi-party collaboration and make decisions about platform support. Shifting between the abstract and concrete models helped to ensure applicability in the concrete case and reflect on its generalisation to other cases with the interviewee, a technique already used by [15]. The discussion of problems and challenges allowed identifying goals to be achieved for the actor represented by the interviewee that could not be achieved without the multi-party collaboration. This technique of goal discovery was used by many other authors [7], [13], [16], [20], [27]. Alternative goal refinements allowed discussing trade-offs of different strategies for goal achievement, a technique already used by [13] and [24]. The trade-off discussions were complemented with discussions about what should be in-scope for the platform support and what may be excluded from the scope, a technique used [13] who differentiated between the intra-, inter-, and extra-organisational domains. Techniques that we did not use were checklists (used by [18]) and analysis of reachability (used by [23]). Instead, we 
used inconsistencies detected in the diagrams that overlapped in content to drive the discussion towards a harmonised documentation of the multi-party collaboration case. To mitigate the issue of harmonising updates between the different characterisation levels, a basic approach will be to define a glossary of terms. Once tool support for our framework is available, an extension with the crosscutting modelling approach of the Adora [49] models can be considered.

\section{VIII.SUMMARY AND CONCLUSIONS}

This paper proposed a framework for the analysis of multiparty collaboration that supports platform developers in analysing future ecosystems that should be supported with a platform. The framework is based on a synthesis of previous approaches to modelling collaboration for requirements engineering. The paper presented an early validation of the framework by describing its application on a case of multi-party collaboration that is to be supported by a platform enabling collaborative development of AI systems. It showed that the framework was useful to identify requirements, especially when the modelling was supported by the analysis tactics of shifting between the abstract and concrete, discussing problems and challenges, reasoning about alternative goal refinements, and decision-making about platform scope. Future research will focus on a more comprehensive validation of the framework by involving all platform stakeholders, considering heterogeneous cases that are to be supported by the platform, and exploring replication.

\section{ACKNOWLEDGEMENT}

This project has received funding from the European Union's Horizon 2020 research and innovation programme under grant agreement No 732204 (Bonseyes). This work is supported by the Swiss State Secretariat for Education, Research and Innovation (SERI) under contract number 16.0159. The opinions expressed and arguments employed herein do not necessarily reflect the official views of these funding bodies.

\section{REFERENCES}

[1] M. Ringel, F. Grassl, R. Baeza, D. Kennedy, M. Spira, and J. Manly, "The Most Innovative Companies 2019: Rise of AI, Platforms, and Ecosystems." [Online]. Available: https://www.bcg.com/publications/collections/most-innovativecompanies-2019-artificial-intelligence-platformsecosystems.aspx. [Accessed: 08-Apr-2019].

[2] M. W. Van Alstyne, G. G. Parker, and S. P. Choudary, "Pipelines, platforms, and the new rules of strategy," Harvard business review, vol. 94, no. 4, pp. 54-62, 2016.

[3] T. Llewellynn et al., "BONSEYES: Platform for Open Development of Systems of Artificial Intelligence: Invited paper," in CF'17 Proceedings of the Computing Frontiers Conference, Siena, Italy, 2017, pp. 299-304.

[4] S. Jansen, S. Brinkkemper, and A. Finkelstein, "Business Network Management as a Survival Strategy: A Tale of Two Software Ecosystems.," in Proceedings of the First International Workshop on Software Ecosystems ( IWSECO-2009), 2009, vol. 09/2009.
[5] F. Fotrousi, S. A. Fricker, M. Fiedler, and F. Le-Gall, "Kpis for software ecosystems: A systematic mapping study," presented at the International Conference of Software Business, 2014, pp. 194-211.

[6] S. Jansen, "Measuring the health of open source software ecosystems: Beyond the scope of project health," Information and Software Technology, vol. 56, no. 11, pp. 1508-1519, 2014.

[7] E. Knauss, A. Yussuf, K. Blincoe, D. Damian, and A. Knauss, "Continuous clarification and emergent requirements flows in open-commercial software ecosystems," Requirements Engineering, vol. 23, no. 1, pp. 97-117, Mar. 2018.

[8] P. Abrahamsson, J. Bosch, S. Brinkkemper, and A. Mädche, "Software Business, Platforms, and Ecosystems: Fundamentals of Software Production Research," Leibniz Center for Informatics, Dagstuhl, Seminar 18182, 2018.

[9] T. Hong, P. Pinson, and S. Fan, "Global Energy Forecasting Competition 2012," International Journal of Forecasting, vol. 30, no. 2, pp. 357-363, Apr. 2014.

[10] M. Ilich, B. Becerik, and B. Aultman, "Online Collaboration: Why Aren't We Using Our Tools?," The Construction Zone, vol. 6, no. 3, 2006.

[11] R. W. Hobbs, "Leadership through collaboration," AIArchitect, vol. 3, no. 11, 1996.

[12] L.-M. Priego-Roche, D. Rieu, and A. Front, "A $360^{\circ}$ Vision for Virtual Organizations Characterization and Modelling: Two Intentional Level Aspects," in Software Services for e-Business and e-Society, vol. 305, C. Godart, N. Gronau, S. Sharma, and G. Canals, Eds. Berlin, Heidelberg: Springer Berlin Heidelberg, 2009, pp. 427-442.

[13] L. M. Priego-Roche, A. Front, and D. Rieu, "A framework for virtual organization requirements," Requirements Engineering, vol. 21, no. 4, pp. 439-460, Nov. 2016.

[14] B. Kitchenham and S. Charters, "Guidelines for performing systematic literature reviews in software engineering," Technical report, Ver. 2.3. EBSE, 2007.

[15] R. A. Buchmann and D. Karagiannis, "Modelling mobile app requirements for semantic traceability," Requirements Engineering, vol. 22, no. 1, pp. 41-75, Mar. 2017.

[16] E. Alreshidi, M. Mourshed, and Y. Rezgui, "Requirements for cloud-based BIM governance solutions to facilitate team collaboration in construction projects," Requirements Engineering, vol. 23, no. 1, pp. 1-31, Mar. 2018.

[17] S. Garde and P. Knaup, "Requirements engineering in health care: the example of chemotherapy planning in paediatric oncology," Requirements Engineering, vol. 11, no. 4, pp. 265-278, Sep. 2006.

[18] L. C. Ballejos and J. M. Montagna, "Method for stakeholder identification in interorganizational environments," Requirements Engineering, vol. 13, no. 4, pp. 281-297, Nov. 2008.

[19] M. E. Porter, Competitive strategy: Techniques for analyzing industries and competitors. Simon and Schuster, 2008.

[20] V. Pijpers, P. de Leenheer, J. Gordijn, and H. Akkermans, “Using conceptual models to explore business-ICT alignment in networked value constellations: Case studies from the Dutch aviation industry, Spanish electricity industry and Dutch telecom industry," Requirements Engineering, vol. 17, no. 3, pp. 203-226, Sep. 2012. 
[21] M. Daneva and R. J. Wieringa, "A requirements engineering framework for cross-organizational ERP systems," Requirements Engineering, vol. 11, no. 3, pp. 194-204, Jun. 2006.

[22] Eric. S. K. Yu, "Towards modelling and reasoning support for early-phase requirements engineering," in Proceedings of ISRE '97: 3rd IEEE International Symposium on Requirements Engineering, Annapolis, MD, USA, 1997, pp. 226-235.

[23] M. Gharib, P. Giorgini, and J. Mylopoulos, "Analysis of information quality requirements in business processes, revisited," Requirements Engineering, vol. 23, no. 2, pp. 227249, Jun. 2018.

[24] F. Dalpiaz, P. Giorgini, and J. Mylopoulos, "Adaptive sociotechnical systems: a requirements-based approach," Requirements Engineering, vol. 18, no. 1, pp. 1-24, Mar. 2013.

[25] V. Bryl, P. Giorgini, and J. Mylopoulos, "Designing sociotechnical systems: from stakeholder goals to social networks," Requirements Engineering, vol. 14, no. 1, pp. 47-70, Feb. 2009.

[26] P. Bresciani, A. Perini, P. Giorgini, F. Giunchiglia, and J. Mylopoulos, "Tropos: An agent-oriented software development methodology," Autonomous Agents and Multi-Agent Systems, vol. 8, no. 3, pp. 203-236, 2004.

[27] J. Barata and P. R. da Cunha, "Mending the patchwork of requirements from multiple standards using participative goal modelling: a case in the food industry," Requirements Engineering, vol. 23, no. 4, pp. 425-441, Nov. 2018.

[28] International Telecommunication Union, "ITU-T (2012) User requirements notation (URN): language definition (Z.151, 10/2012)." [Online]. Available: https://www.itu.int/rec/T-RECZ.151/en. [Accessed: 08-Apr-2019].

[29] L. Patrício, J. Falcão e Cunha, and R. P. Fisk, "Requirements engineering for multi-channel services: the SEB method and its application to a multi-channel bank," Requirements Engineering, vol. 14, no. 3, pp. 209-227, Jul. 2009.

[30] L. Chung and J. C. S. do Prado Leite, "On non-functional requirements in software engineering," in Conceptual modeling: Foundations and applications, Springer, 2009, pp. 363-379.

[31] S. Jansen, S. Brinkkemper, and A. Finkelstein, "Providing transparency in the business of software: a modeling technique for software supply networks," presented at the Working Conference on Virtual Enterprises, 2007, pp. 677-686.

[32] J. Gordijn and J. M. Akkermans, "Value-based requirements engineering: exploring innovative e-commerce ideas," Requirements Engineering, vol. 8, no. 2, pp. 114-134, Jul. 2003.

[33] C. Wolter and C. Meinel, "An approach to capture authorisation requirements in business processes," Requirements Engineering, vol. 15 , no. 4, pp. 359-373, Nov. 2010.

[34] A.-W. Scheer, ARIS-business process frameworks. Springer Science \& Business Media, 2012.

[35] J. A. Zachman, The Zachman Framework for Enterprise Architecture: A Primer for Enterprise Engineering and Manufacturing. Zachman International, Inc., 2003.
[36] The Open Group, “The TOGAF® Standard, Version 9.2," Apr2018. .

[37] Z. Derzsi and J. Gordijn, "A Framework for Business/IT Alignment in Networked Value Constellations," in Proceedings of the CAISE*06 Workshop on Business/IT Alignment and Interoperability BUSITAL '06, Luxemburg, 2006, pp. 219-226.

[38] D. Tapscott, D. Ticoll, and A. Lowy, Digital capital: Harnessing the power of business webs. Harvard Business School Press, 2000.

[39] R. J. Wieringa, Design science methodology for information systems and software engineering. Springer, 2014.

[40] T. Gorschek, P. Garre, S. Larsson, and C. Wohlin, "A Model for Technology Transfer in Practice," IEEE Software, vol. 23, no. 6, pp. 88-95, Nov. 2006.

[41] S. E. Hove and B. Anda, "Experiences from Conducting Semistructured Interviews in Empirical Software Engineering Research," in 11th IEEE International Software Metrics Symposium (METRICS'05), Como, Italy, 2005, pp. 23-23.

[42] D. Ostler, N. Marahrens, D. Wilhelm, and H. Feußner, "The Bonseyes-Project: democratizing Artificial Intelligence for the collaborative OR," presented at the Computer Assisted Radiology and Surgery CARS 2019, Rennes, France.

[43] The Object Management Group ${ }^{\circledR}(\mathrm{OMG} \AA)$, “About the Business Process Model And Notation Specification Version 2.0," Jan2011. [Online]. Available: https://www.omg.org/spec/BPMN 12.0/. [Accessed: 30-Mar-2019].

[44] The Object Management Group ${ }^{\circledR}(\mathrm{OMG} \AA)$, “About the Unified Modeling Language Specification Version 2.5.1," Dec-2017. [Online]. Available: https://www.omg.org/spec/UML/. [Accessed: 30-Mar-2019].

[45] P. Runeson and M. Höst, "Guidelines for conducting and reporting case study research in software engineering," Empirical software engineering, vol. 14, no. 2, p. 131, 2009.

[46] S. Nesland, "Initial lessons learned from the definition and implementation of a platform requirements engineering process at Intel Corporation," presented at the 13th IEEE International Conference on Requirements Engineering (RE'05), 2005, pp. 429-433.

[47] L. Liu and E. Yu, "From requirements to architectural designusing goals and scenarios," in First International Workshop From Software Requirements to Architectures-STRAW, 2001, vol. 1, p. 22.

[48] G. Regev and A. Wegmann, "Where do goals come from: the underlying principles of goal-oriented requirements engineering," in 13th IEEE International Conference on Requirements Engineering (RE'05), 2005, pp. 353-362.

[49] S. Meier, T. Reinhard, R. Stoiber, and M. Glinz, "Modeling and evolving crosscutting concerns in ADORA," in Early Aspects at ICSE: Workshops in Aspect-Oriented Requirements Engineering and Architecture Design (EARLYASPECTS'07), 2007. 